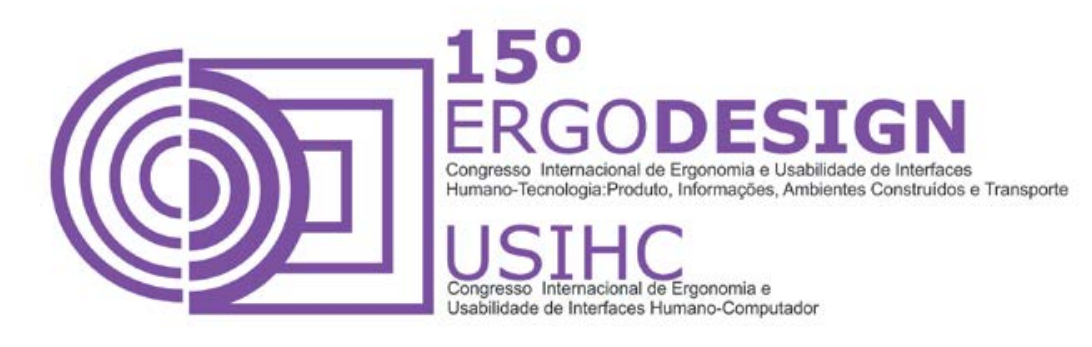

\title{
PRESCRIÇÃO E USO DE ANDADORES PARA IDOSOS: UMA DEMANDA PARA O DESIGN ERGONÔMICO
}

\section{PRESCRIPTION AND USE FOR ELDERLY WALKERS: A CLAIM FOR DESIGN ERGONOMIC}

\author{
BOIANI, Josieli Aparecida Marques (1); \\ FERREIRA, Ana Cristina Maurício (2); \\ BOTURA JUNIOR, G. (3); \\ PASCHOARELLI, Luis Carlos (4); \\ MEDOLA, Fausto Orsi (5). \\ (1) PPGDesign-UNESP/Bauru, Designer \\ e-mail:jboiani@yahoo.com.br \\ (2) PPGDesign-UNESP/Bauru, Doutoranda \\ e-mail:anacr fatec@yahoo.com.br \\ (3) PPGDesign-UNESP/Bauru, Doutor \\ e-mail:galdenoro@sorocaba.unesp.br \\ (4) PPGDesign-UNESP/Bauru, Doutor \\ e-mail:paschoarelli@faac.unesp.br \\ (5) PPGDesign-UNESP/Bauru, Doutor \\ e-mail: fausto.medola@faac.unesp.br
}

\begin{abstract}
RESUMO
O processo de envelhecimento pode levar à redução/restrição da mobilidade pessoal, podendo ser necessário o uso de dispositivos de Tecnologia Assistiva. O objetivo deste estudo foi discutir os aspectos ergonômicos relacionados à prescrição e uso de andadores. Uma revisão bibliográfica foi realizada e os resultados apontam que a prescrição dos dispositivos nem sempre é adequada, o que gera frequente uso inadequado e consequente abandono do uso do equipamento. Também constatou-se uma ampla demanda para investigações na área do design ergonômico, visto o pequeno número de pesquisas relacionados diretamente aos aspectos de design e ergonomia do produto.
\end{abstract}

Palavras-chave: Envelhecimento populacional. Andadores. Mobilidade reduzida. 


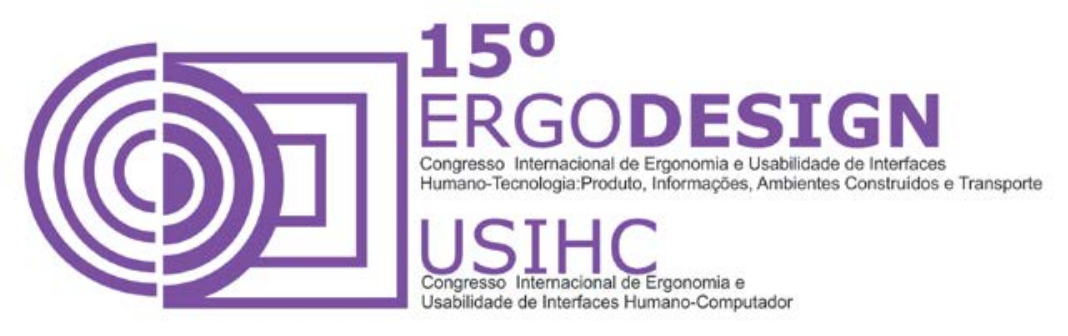

\begin{abstract}
Some implications of the elderly health lead to a reduction on the mobility and poor quality of life. This requires the assistance of caregivers and/or the use of assistive technology devices. This study aimed to discuss the ergonomic aspects of prescription and usage of walkers. A literature review was carried out and the results show that the prescription is not always adequate, what may cause inappropriate use and, consequently, equipment abandonment. The study revealed a large demand on research in the field of design and ergonomics, due to the relatively few number of papers directly related to the ergonomic aspects of the design of walkers.
\end{abstract}

Keywords: Elderly. Walkers. Reduced mobility.

\title{
1. INTRODUÇÃO
}

O número de idosos no Brasil tem crescido substancialmente nas últimas décadas. Isto se deve às alterações dos índices demográficos: aumento na expectativa de vida, redução da taxa de mortalidade e da taxa de fecundidade. Essas alterações se traduzem em diminuição do número de crianças e aumento da população idosa (OPAS, 2005).

Diferentes implicações na saúde e bem estar afetam o ciclo de vida e o perfil desta faixa populacional (Brito; Litvoc, 2004). Os idosos podem ser saudáveis, especialmente no caso daqueles indivíduos que mantém práticas de atividades físicas, entre outros inúmeros fatores (Shephard, 2003). Mas, de modo geral, isto não ocorre, pois os problemas de saúde típicos das idades mais avançadas surgem restrições mais expressivas na queda da qualidade de vida causadas pelo acúmulo dos efeitos do envelhecimento (Birren; Schroots, 1996).

Os principais fatores que levam ao decréscimo na qualidade de vida do idoso são a redução da capacidade cardíaca (Spirduso,2002; Affiune, 2002), a redução da capacidade respiratória (De Vitta, 2000; Gorzoni e Russo, 2002), a perda de massa muscular e óssea (Janssen et al., 2000; Reeves et al., 2003), aumento da pressão arterial e sistólica (Shephard, 2003), comprometimento do sistema nervoso central (Cançado e Horta, 2002), entre outros. De ordem psicológica são citadas as ocorrências de depressão e ansiedade (Zimerman, 2000).

Todos estes fatores levam os indivíduos a apresentar declínio em sua qualidade de vida e em sua mobilidade, aumentando os riscos de ocorrência de quedas, diminuindo a facilidade de mobilidade e, enfim, apresentando dependência e prejuízo em sua autonomia. Estas limitações impostas pelo processo de envelhecimento levam ao idoso necessitar do auxílio de terceiros, sejam eles cuidadores, enfermeiros ou familiares ou o uso de dispositivos de Tecnologia Assistiva para a realização das atividades da vida diária, buscando segurança e melhora na qualidade de vida (Reeves et al., 2003; Schneider et al., 2008).

Dos dispositivos pertencentes à Tecnologia Assistiva, os de auxílio à mobilidade são aqueles que alternam ou complementam a mobilidade. Os dispositivos alternativos à mobilidade, como as cadeiras de rodas, são direcionados às pessoas com incapacidade total de mobilidade. Já os dispositivos complementares à mobilidade são aqueles que auxiliam a marcha do indivíduo com mobilidade reduzida, como as bengalas, as muletas e os andadores. De forma geral, estas tecnologias devolvem ao idoso a autonomia e independência para a realização das tarefas rotineiras e participação social, imprescindíveis para a percepção da qualidade de vida e do 


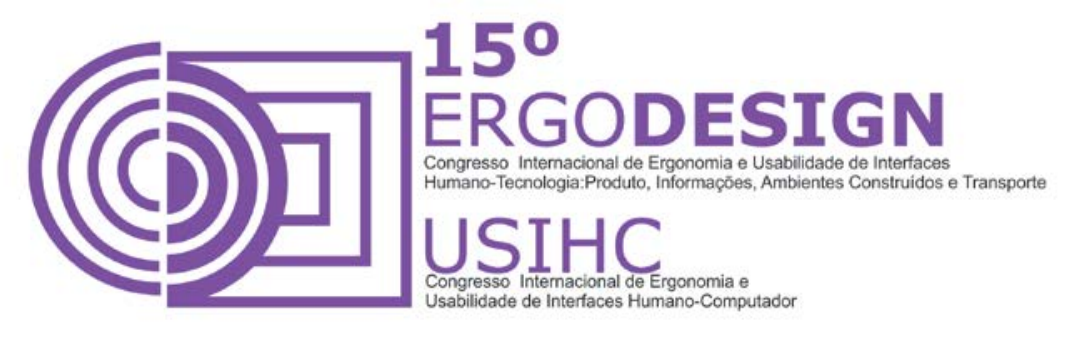

bem estar. Por outro lado, nota-se que o design destes dispositivos complementares apresenta elevada demanda para desenvolvimento e aperfeiçoamento tecnológico e estético, particularmente ao que refere a relação entre prescrição e uso dos andadores, o que poderia ampliar a qualidade geral dos mesmos e a usabilidade dos idosos (Bersch, 2013).

O objetivo deste estudo foi discutir os aspectos ergonômicos relacionados à prescrição e uso de dispositivos complementares à mobilidade, particularmente os andadores, por meio de revisão de estudos científicos.

\section{REVISÃO DE LITERATURA}

\subsection{O processo de envelhecimento: implicações na mobilidade}

No Século XX o Brasil apresentou, de forma bastante expressiva, uma alteração em sua demografia. Se em décadas anteriores a 1960 o país tinha uma população essencialmente constituída por jovens, a partir deste momento a formação demográfica começou a passar por uma revolução, com o crescente número de idosos e a diminuição do número de crianças (OPAS, 2005). Basicamente, os fatores contribuintes para o envelhecimento da população, conforme citado por Minayo (2012) são o aumento da expectativa de vida, a redução da taxa de fecundidade e a redução da taxa bruta de mortalidade. Esta alteração no quadro demográfico acarreta em implicações em todo o ciclo de vida e perfil da população.

Conforme observado por Netto (2004), a população de idosos nem sempre atinge esta etapa da vida de forma plenamente satisfatória, surgindo os problemas de saúde, que podem ser de ordem psicológica ou organofuncional, afetando principalmente os menos favorecidos economicamente.

Quanto às implicações psicológicas, Canineu e Bastos (2002) citam o processo de rememoração e a constante dificuldade para lembrar nomes e locais de objetos guardados. Assis e Araújo (2004) alerta sobre o aumento da ocorrência de depressão e ansiedade; e Spirduso e Cronin (2001) descrevem a incidência de depressão e também o surgimento do declínio cognitivo em idosos. Para Teixeira (2004), sentimentos como angustia e quadros depressivos estão associados às decadências físicas.

Da ordem organofuncional, as principais implicações observadas dizem respeito à diminuição da ventilação pulmonar, aumento da pressão arterial sistólica, perda de massa muscular, perda de massa óssea, diminuição da elasticidade dos tecidos conectivos, perda parcial dos líquidos sinoviais, diminuição da flexibilidade de tendões e ligamentos e comprometimento do sistema nervoso central.

Hayflick (1997), De Vitta (2000), Affiune (2002), Gallahue e Ozmun (2005) abordaram em seus estudos, o declínio da eficácia aeróbica com o avanço da idade, pois o envelhecimento acarreta a diminuição da ventilação pulmonar, reduzindo a elasticidade dos alvéolos e subtraindo a capacidade vital. Conforme aborda Shephard (2003), o envelhecimento é acompanhado do aumento da pressão arterial sistólica, ocorrendo uma crescente hipotensão postural resultante de uma regulação deficiente da pressão arterial. 


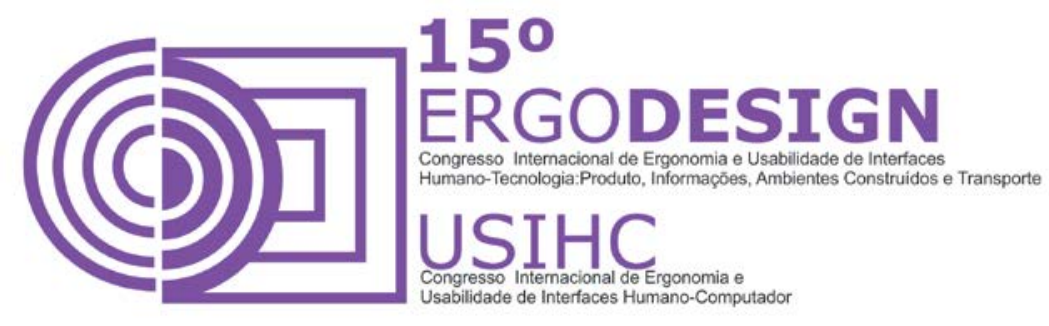

Janssen et al. (2000), Shephard (2003), Reeves et al (2003), afirmam que a maior implicação do envelhecimento está no comprometimento da força muscular, que ocorre devido ao enrijecimento dos seus tendões. Asseguram que o declínio da massa muscular, que tem início por volta dos cinqüenta anos de idade, leva a uma perda crescente da força e da resistência aeróbia. Matsudo, Matsudo e Barros (2000), descrevem que entre os 25 e 65 anos, acontece uma depreciação de $10 \%$ a $16 \%$ na massa muscular magra. Para eles, esta decrescente redução ocorre devido ao envelhecimento da massa óssea no músculo esquelético, e em razão da diminuição da água no corpo. De Vitta (2000), afirma que as modificações tornam-se evidentes com o envelhecimento do sistema músculo esquelético, ocorrendo a diminuição no comprimento, na elasticidade e no número de fibras. Também cita a perda de massa muscular, a perda de parte do líquido sinovial e da elasticidade dos tecidos conectivos.

Weineck (1991) relata como prevalência importante no envelhecimento a perda de massa óssea. Todos os fatores mencionados levam a um prejuízo organofuncional, acometendo a qualidade de vida do idoso. A diminuição na flexibilidade dos tendões e ligamentos, de acordo com Shephard (2003) e Reeves et al (2003) leva à ocorrência de torções, luxações e aumento do risco de quedas. Schneider et al (2008), relatam que aproximadamente um terço da população idosa sofre quedas, crescendo a frequência com o aumento da idade.

Para autores como Cançado e Horta (2002) e De Vitta (2000), o sistema nervoso central, responsável por funções biológicas internas, sensações, movimentos e funções psíquicas, é o mais afetado pelo envelhecimento, apresentando alterações como a redução do número de neurônios, a diminuição da velocidade de condução nervosa e a lentidão dos reflexos.

Os fatores correlacionados ao processo de envelhecimento levam o idoso à redução significativa de sua qualidade de vida, aumentando o risco de queda, dificultando a mobilidade pessoal e diminuindo a autonomia na realização de tarefas da vida diária. Todos os autores são enfáticos quanto à realização de exercícios físicos, que ajudam a melhorar a saúde psicológica, a função muscular, a eficiência cardíaca, a função pulmonar, diminuindo a perda de massa muscular e prevenindo a perda de massa óssea. Assim, de acordo com Cress et al (1999), as pessoas que se mantêm ativas fisicamente no decorrer da vida, mostram vantagens em sua saúde quando em idade avançada, apresentando maior autonomia e independência.

Por outro lado, na maioria das vezes, os indivíduos deixam de manter suas atividades físicas com o avanço da idade, o que leva a uma ampla demanda para o uso de dispositivos de auxílio à mobilidade.

\subsection{Dispositivos de auxílio à mobilidade}

Diversas são as razões que levam uma pessoa a necessitar de dispositivos que a auxiliem na marcha ou na mobilidade, seja de forma temporária ou permanente. A necessidade de locomoção é intrínseca ao ser humano e, se por alguma razão, torna-se limitada, pode-se recorrer a dispositivos que o auxiliem nesta tarefa. São dispositivos desenvolvidos exclusivamente para um usuário ou, de forma mais comum, para um determinado público. De acordo com Berschi (2013), a mobilidade pode ser auxiliada por bengalas, muletas, andadores, carrinhos, cadeiras de rodas manuais ou elétricas, scooters e qualquer outro veículo, equipamento ou estratégia utilizada na melhoria da mobilidade pessoal. 


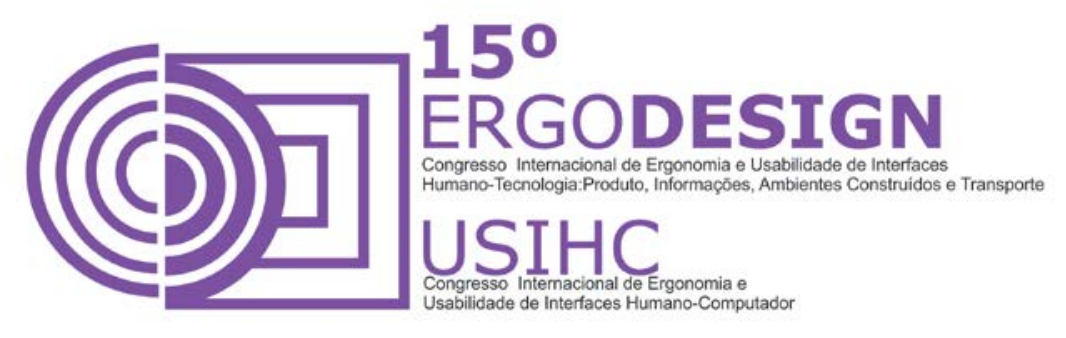

Os dispositivos de auxílio à mobilidade podem ser entendidos como aqueles que agem como alternativa ou como complemento à mobilidade. Em caso de incapacidade total da mobilidade, são utilizados dispositivos que agem como alternativa ao ato de locomoção ou mobilidade, como as cadeiras de rodas de propulsão manual, motorizadas ou scooters. Quanto às cadeiras de rodas, diferentemente de sua classificação habitual quanto ao uso, Bertoncello e Gomes (2002) diferenciam o dispositivo quanto ao grau de tecnologia empregado, podendo ser de baixa, média ou alta complexidade.

O que distingue a complexidade das cadeiras neste contexto é a participação do designer no projeto. Em um produto de baixa complexidade todos os componentes podem ser solucionados e projetados pelo mesmo profissional. No produto de média complexidade existe a necessidade de envolvimento de profissionais de outras áreas para solucionar problemas específicos e no de alta complexidade o designer irá solucionar apenas alguns componentes do dispositivo, agindo mais na abordagem conceitual e metodológica (Barroso Neto, 1982).

No grupo de tecnologia de baixa complexidade encontram-se as cadeiras de rodas mecanomanuais, que são conduzidas pelo trabalho muscular do usuário ou de uma segunda pessoa. No grupo de média complexidade as eletromecânicas, conhecidas como motorizadas, estando incluídos os triciclos elétricos (scooters). No nível de alta complexidade, as cadeiras de rodas eletroeletrônicas, que possuem componentes eletrônicos e envolvem princípios computacionais.

Para os dispositivos de mobilidade que complementam a marcha, destinados às pessoas com mobilidade reduzida ou, como é o caso de idosos, com deambulação insegura, estão as bengalas, as muletas e os andadores, que ajudam a melhorar a postura e o equilíbrio, adequando a aplicação de carga nos membros inferiores e superiores. Garcia (2014) esclarece que, basicamente, existem três tipos de bengalas, as standard, as em "T" e as de base larga. As bengalas standard possuem apoio curvo para as mãos e são indicadas para pessoas que devem poupar algum membro da descarga de peso ou que possuem algum fator que provoque instabilidade corporal. As bengalas em "T", apesar da mesma indicação da standard, têm como diferencial sua extremidade superior possuir o formato da letra T, o que proporciona maior segurança às pessoas com força reduzida nas mãos. Por fim, as bengalas de base larga podem ter três ou quatro apoios, o que permite maior nível de equilíbrio e segurança.

Conforme Brumini (2014), as muletas são utilizadas para aumento do equilíbrio e para o alívio total ou parcial dos membros inferiores e tem como funções aumentar a base de sustentação e melhorar a estabilidade lateral, permitindo que os membros superiores transfiram o peso corporal para o solo. Classificam-se em axilares e canadenses. Kaempf (2014) alerta que as muletas axilares, apesar do baixo custo de aquisição, devem ser utilizadas de forma adequada, sendo que no momento da deambulação a força deve ocorrer nos cotovelos e punhos e não nas axilas, de modo a evitar desconforto ou lesões. As muletas canadenses têm como apoio a existência de braçadeira para o antebraço, podendo ser utilizadas por pessoas com bom equilíbrio corporal e membros superiores fortes. Garcia (2014) salienta que a vantagem da muleta canadense é a possibilidade de realização de atividades da vida diária, sem que as muletas se soltem. 


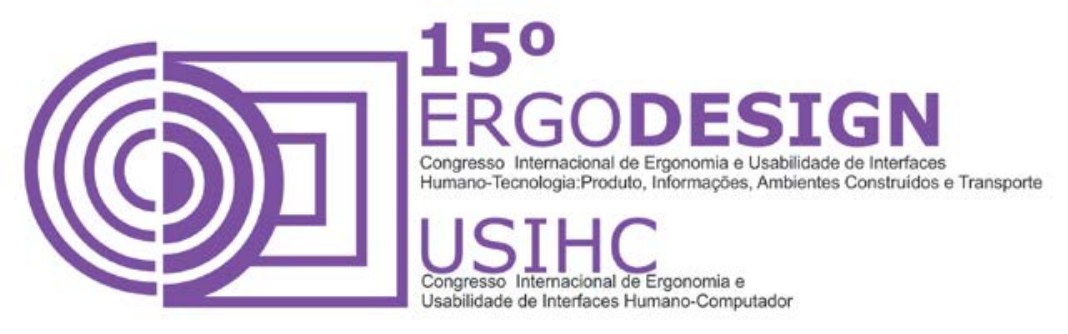

Os andadores, assim como as bengalas e as muletas, são dispositivos de tecnologia assistiva para o auxílio à marcha que, por ter uma base mais ampla de contato com o solo, oferecem apoio eficaz à postura ortostática e ao movimento de marcha, melhorando a estabilidade corporal. De acordo com o Catálogo Nacional de Produtos de Tecnologia Assistiva (BRASIL, 2014), os modelos variam, basicamente, entre pés fixos, com rodízios nos pés anteriores, com assento e com colete de sustentação.

Lee et al. (2014) destaca que o andador é um dispositivo utilizado pelo idoso que precisa de apoio adicional para manter o equilíbrio e a estabilidade ao caminhar, mas que, no entanto apresenta desvantagens quanto à existência de obstáculos como tapetes e pisos irregulares. Bateni e Maki (2005) observam que somente nos Estados Unidos, quase dois milhões de pessoas idosas ou com diferentes condições clínicas utilizam andadores para manter 0 equilíbrio e mover-se de forma independente e, que estes dispositivos de auxílio à marcha ajudam a reduzir a carga dos membros inferiores, aliviando as dores nestas articulações e compensando a fraqueza ou lesão do usuário. Os dispositivos devem ser adequadamente prescritos e periodicamente revisados, verificando a necessidade ou a alteração desta, de forma a continuar propiciando a segurança e eficácia.

\subsection{O andador como recurso de tecnologia assistiva para o idoso}

Com as limitações que o processo de envelhecimento por vezes impõe ao ser humano, recursos tecnológicos são empregados no intuito de promover uma melhora na qualidade de vida do idoso. Andrade e Pereira (2009) descrevem que há três formas de envelhecimento: a comum, a saudável e a fragilizada. Nesta última os idosos apresentam-se debilitados e dependentes de terceiros.

Os recursos empregados que visam auxiliar na realização das tarefas do idoso e, por vezes, restaurar seu nível de autonomia, dividem-se em existência de cuidadores, uso de cadeiras de rodas, bengalas, muletas e andadores. Além dos tipos mais comuns de andadores, de pés fixos (Figura 1a) e com rodízios nos pés anteriores (Figura 1b), são comercializados no Brasil modelos com assentos (Figura 1c), indicados para o auxílio à marcha e para descanso e os andadores com coletes de sustentação (Figura 1d) destinado à elevação do tronco para fins de realização do movimento de marcha.

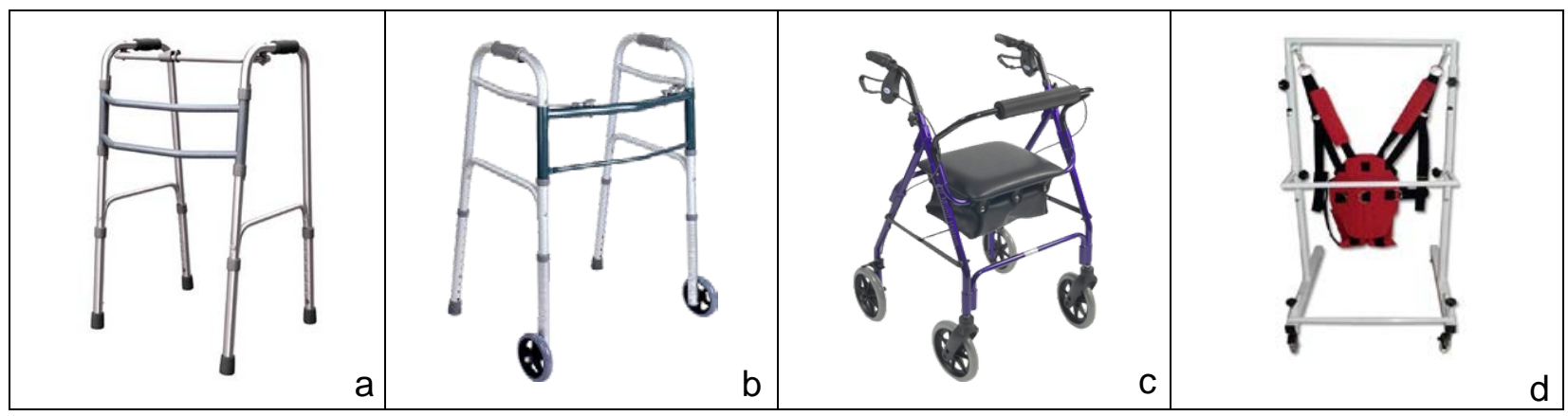

Figura 1 - Andadores comumente comercializados no Brasil Fonte: MCTI, 2014 


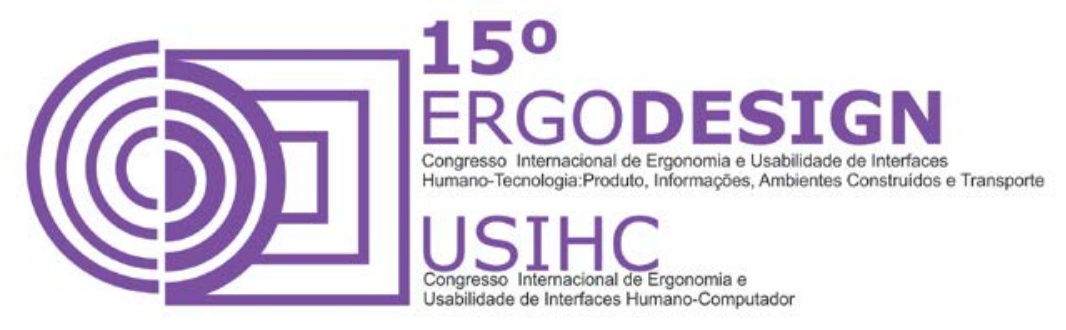

No exterior, outros modelos podem ser encontrados, como o drive rollator (Figura 2a), que apresenta rodízios nos quatro pés, freios, encosto removível e assento, o standers metro Walker (Figura 2b), que é dobrável, o u-step Walker 2 (Figura 2c), projetado para pessoas com variados tipos de privações e o drive bariatric one arm side Walker (Figura 2d), concebido para a utilização de apenas uma mão ou braço como apoio.

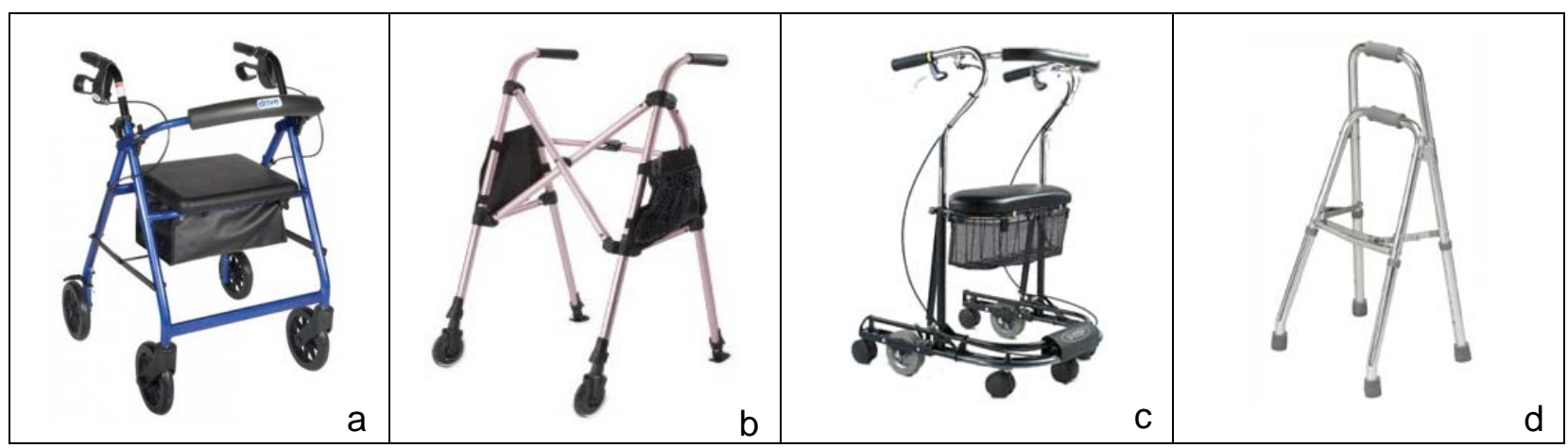

Figura 2 - Andadores comercializados no exterior

Fonte: http://www.1800wheelchair.com

Andrade e Pereira (2009) observam que vários tipos de tecnologias assistivas são implementados no intuito de promover aos idosos, fragilizados ou não, uma vida independente, com capacidade de realização de tarefas. Mas no Brasil há carência no conhecimento e aquisição destes dispositivos de auxílio à mobilidade, principalmente nas classes sociais de baixa renda. Muitos destes usuários adquirem os dispositivos sem prescrição feita por profissional da saúde, diretamente em farmácias, lojas de produtos ortopédicos ou de outros usuários que deixaram de fazer uso. Esta situação gera preocupação, pois a aquisição sem a prescrição adequada não leva em consideração a patologia ou necessidade do usuário e as características do dispositivo, podendo acarretar outros problemas físicos como desconforto, dores ou lesões. Se prescritos adequadamente, têm o potencial de melhorar a independência e qualidade de vida do idoso.

\subsection{Características do uso de andadores em idosos}

Conforme descreve César (2010), a capacidade funcional pode ser entendida como a capacidade de manter as habilidades físicas e mentais necessárias para a manutenção de vida independente e com autonomia, assim a perda da capacidade funcional compromete a autonomia, determinando vários graus de dependência. Afirma ainda que, a existência das limitações físicas, cognitivas ou sensoriais não leva necessariamente à dificuldade de realização das atividades diárias, devido ao avanço de tecnologias assistivas, como os dispositivos de apoio ou adequação ambiental.

Apesar das limitações financeiras e a constante falta de prescrição profissional, Thomas et al. (2008) analisa o uso dos andadores prescritos no desempenho funcional de idosos, entendendo que existe a necessidade de profissionais de reabilitação a fim de verificar as necessidades pessoais, o desempenho e os fatores ambientais, otimizando a adoção do andador na vida 


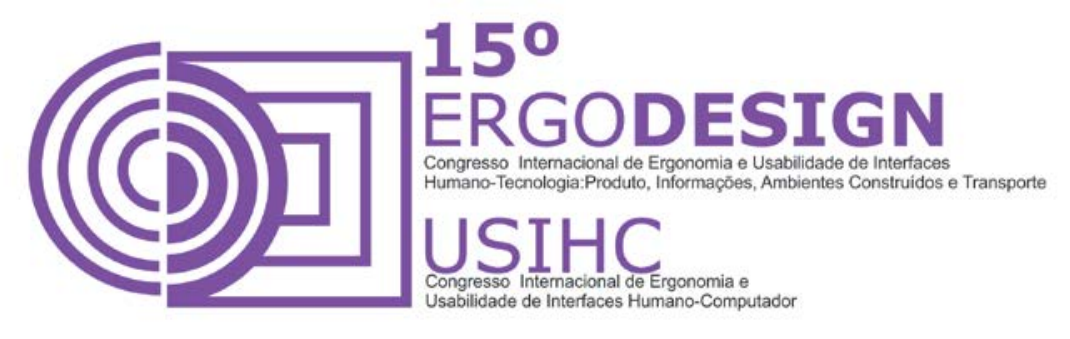

diária dos idosos. Mann et al (1995) em pesquisa realizada nos Estados Unidos com usuários de andadores, relatam elevados casos de usuários que encontram problemas em seu uso, muitos classificando o dispositivo como de difícil uso ou perigoso. Aponta também para a necessidade de uma rigorosa avaliação, prescrição e acompanhamento profissionais.

De acordo com Tomita et al. (2004), os andadores, são utilizados para manter a independência e permitir que os idosos realizem as tarefas da vida diária. Dentro deste princípio, os autores analisam as necessidades que levam à prescrição do dispositivo, sendo a principal o nível de dependência física, seguidas por ingestão de medicamentos e ocorrência de variações psicossociais como a depressão. Freedman et al. (2005) descrevem a tendência entre os americanos idosos não institucionalizados, que a partir da década de 90 vem, gradativamente, diminuindo a necessidade de cuidadores e aumentando o uso dos dispositivos de apoio, o que os confere maior autonomia. Para Agree e Freedman (2011), os dispositivos de apoio à mobilidade utilizados pelos idosos propiciam maior qualidade de vida em usuários que apresentam os maiores níveis de limitações físicas.

O estudo realizado por Lin e Wu (2014) analisa as associações entre limitações na mobilidade, o uso de andadores ou o auxílio de cuidadores, considerando o bem estar e a qualidade de vida do usuário. Foi concluído que o auxílio realizado pelos cuidadores afetam negativamente os idosos, enquanto o uso de dispositivos de apoio tem aspectos positivos quanto à realização eficaz das atividades e, consequentemente, a melhora na qualidade de vida. Mello e Ramos (1999) afirmam que idosos dependentes podem apresentar grande necessidade de uso de dispositivos de apoio, concluindo que a demanda de serviços de reabilitação para idosos que incluem o processo de prescrição destes equipamentos, não são atendidas plenamente em nosso país, carecendo de desenvolvimento nesta área. Ainda, relatam que grande parte das aquisições de andadores são indicações não especializadas, geralmente por amigos ou familiares, reforçando a tendência à automedicação e o aumento ao risco de quedas. Quando os idosos têm a prescrição de um profissional, por vezes relatam a negligência dos mesmos quanto às especificidades pessoais.

Souza et al. (2011) buscaram conhecer as representações sociais sobre Tecnologias Assistivas, e destacam que os idosos apresentam dificuldades no uso dos dispositivos de apoio devido a problemas relacionados à cognição ou por razões culturais. Relatam a frustração apresentada pelos idosos em ter a necessidade de uso dos dispositivos evidenciada, o que para eles atestam suas limitações físicas e sociais. Também citam a baixa qualidade estética, 0 incômodo na realização das tarefas e a má qualidade dos materiais empregados, o que leva à rápida necessidade de manutenção. Por fim, relatam a instrução e a prescrição deficientes por parte dos profissionais envolvidos, levando ao desconhecimento das vantagens por parte do idoso.

Andrade e Pereira (2009) consideram que os problemas de saúde presentes no processo de envelhecimento, devem-se frequentemente ao declínio funcional do indivíduo. $O$ uso de tecnologias assistivas ajudam o idoso a realizar as atividades funcionais da vida diária com segurança, aumentando sua independência e autonomia, prevenindo comorbidades e contribuindo para melhora na qualidade de vida. 


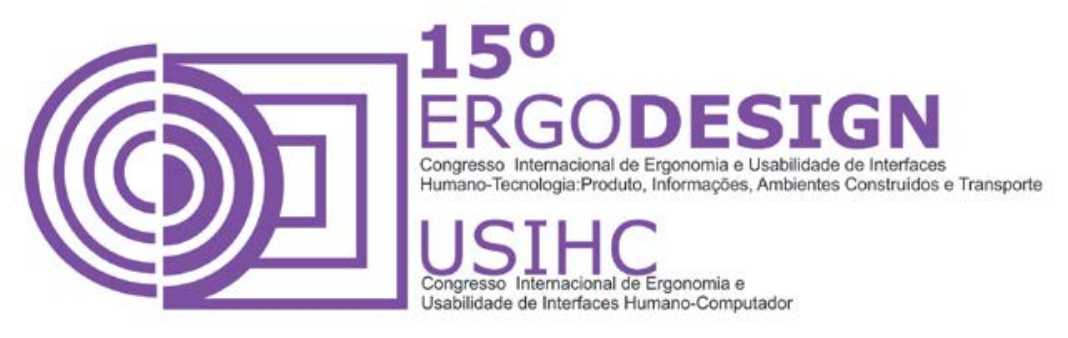

\section{CONSIDERAÇÕES FINAIS}

O envelhecimento, em grande parte dos casos, ocorre de maneira pouco saudável, com implicações na saúde física e psicológica do idoso, levando ao surgimento de necessidades especiais para manter sua mobilidade e segurança. Este aspecto observado no estudo destacase em países menos desenvolvidos (como é o caso do Brasil) e, por consequência, em populações menos favorecidas economicamente.

Os fatores que levam ao declínio da qualidade de vida dividem-se em psicológicos e organofuncionais. Neste último caso, a qualidade de vida na terceira idade também é influenciada pela baixa realização de atividades físicas, as quais são fundamentais para a integridade física, funcional e psicológica do indivíduo, podendo restaurar funções em declínio no idoso. O uso do andador, neste sentido, auxilia não somente na realização das atividades da vida diária e na participação social, mas principalmente na possibilidade do idoso em manter-se ativo.

Em caso da necessidade do uso dos dispositivos de auxílio à mobilidade em nível nacional, existem características negativas quanto à participação eficaz dos profissionais das áreas correlacionadas na prescrição e na orientação aos idosos do uso de tais tecnologias. A prescrição dos andadores pelos profissionais nem sempre considera os aspectos funcionais do usuário. Além disto, a orientação é precária no sentido de informar corretamente as vantagens e os benefícios dos dispositivos de apoio ao idoso.

O equívoco dos profissionais na imprecisa prescrição aliado à falta de informação sobre os benefícios e, por vezes, à má qualidade dos materiais empregados levam o idoso, em quantidade significativa, a renunciar ao uso dos andadores. O não uso também tem forte relação com os fatores psicológicos do usuário, que vê o dispositivo como uma afirmação de sua incapacidade física parcial. Quanto à indicação do dispositivo, esta é comumente realizada por amigos ou familiares dos idosos, contribuindo para o abandono do uso do dispositivo e para o reforço à automedicação.

Se devidamente prescrito e adaptado ao idoso, os dispositivos de auxílio à mobilidade apresentam inúmeros benefícios, como a manutenção da atividade física, a possibilidade de realização pelo idoso das atividades rotineiras e a possibilidade de participação social na comunidade por ele freqüentada. Aumentam os níveis de segurança, independência e autonomia, resultando em melhora na saúde psicológica e na qualidade de vida dos usuários.

Por outro lado, a realização de prescrições associadas à observação das necessidades (física, psicológicas e sociais) dos idosos, deve gerar maior demanda para o aperfeiçoamento do design dos produtos e sistemas, exigindo maior integração entre fatores humanos (ergonômicos) e tecnológicos (de projeto).

Por fim, conclui-se que ainda falta muito para se desenvolver produtos de Tecnologia Assistiva - especialmente, andadores - baseados em conceitos de design e ergonomia, os quais visam a adequação dos produtos e sistemas às necessidades e expectativas dos usuários, sendo que a observação mais importante feita a partir deste estudo é a fragilidade das pesquisas científicas nacionais que abordam o tema em questão, uma vez que as mesmas ainda deixam de considerar a importância do Design Ergonômico nesta área. No âmbito nacional, os 


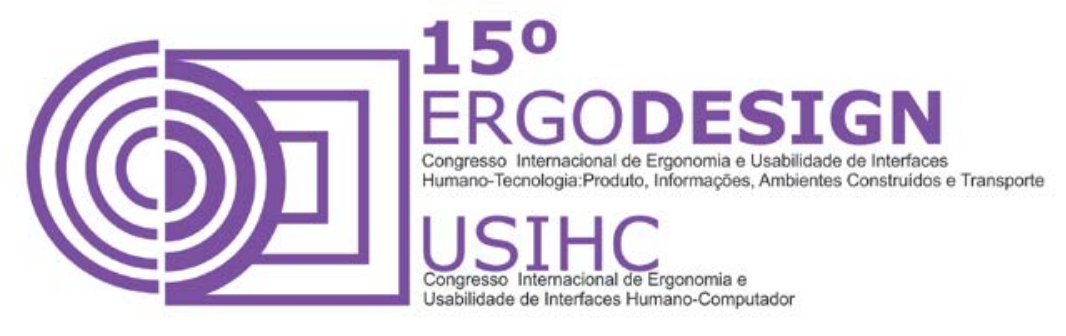

investimentos econômicos em pesquisas científicas não são insuficientes, mas são insuficientes os investimentos intelectuais focados na população idosa, havendo grande necessidade de pesquisas científicas e acadêmicas que envolvam a população idosa e o uso das tecnologias assistivas disponíveis.

\section{REFERÊNCIAS}

AFFIUNE, A. Envelhecimento cardio vascular. Tratado de Geriatria e Gerontologia. Rio de Janeiro: Guanabara Koogan, 2002.

AGREE, E.M.; FREEDMAN, V.A. A quality-of-life scale for assistive technology: results of a pilot study of aging and technology. Jornal of the American Physical Therapy Association. 2011.

ANDRADE, V.S.; PEREIRA, L.S.M. Influência da tecnologia assistiva no desempenho funcional e na qualidade de vida de idosos comunitários frágeis: uma revisão bibliográfica. Revista Brasileira de Geriatria e Gerontologia. 2009.

ASSIS, M.; ARAÚJO, T.D. Atividade e postura corporal. In A.L. Saldanha e, C.P. Caldas (Ed), Saúde do Idoso: A Arte de Cuidar. 2004.

BARROSO NETO, E. Desenho Industrial - Desenvolvimento de Produto. Brasília: CNPq/Coordenação Editorial, 1982.

BATENE, H.; MAKI, B.E. Assistive devices for balance and mobility: benefits, demands and adverse consequences. Physical Medicine and Rehabilitation. 2005.

BERSCH, R. Introdução à tecnologia assistiva. 2013. Disponível em <http://www.assistiva.com.br/>. Acesso em 18 dezembro 2014.

BERTONCELLO, I.; GOMES, L.V.N. Análise diacrônica e sincrônica da cadeira de rodas mecanomanual. Revista Produção, v. 12, n. 1, São Paulo, 2002.

BRASIL, Ministério da Ciência, Tecnologia e Inovação. Catálogo Nacional de Produtos de Tecnologia Assistiva. 2014.

BRITO, F.C; LITVOC, C. J. Conceitos básicos. In F.C. Brito e C. Litvoc (Ed.), Envelhecimento prevenção e promoção de saúde. São Paulo: Atheneu, 2004.

BIRREN, J.E.; SCHROOTS, J.J.F. History, concepts and theory in the psychology of aging. In J.E. Birren

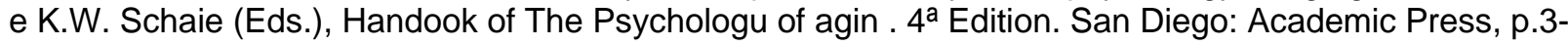
23, 1996. 


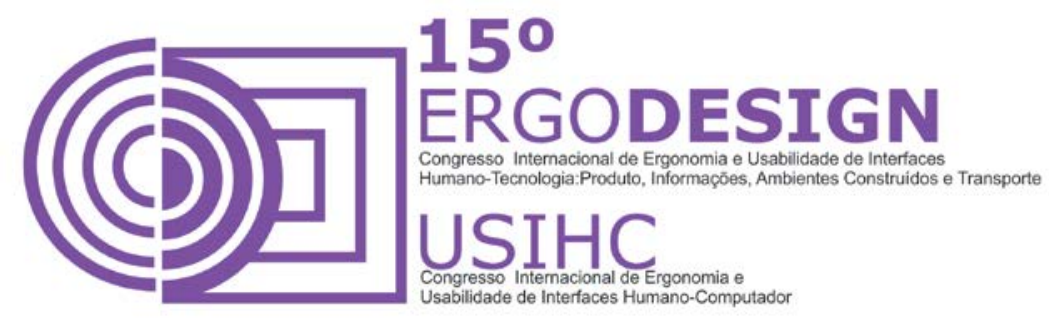

BRUMINI, C. Intervenção Fisioterapeutica. 2014. Disponível em <http://sis.posugf.com.br/>. Acesso em 22 dezembro 2014.

CANÇADO, F.A.X.; HORTA, M.L. Envelhecimento cerebral. Tratado de Geriatria e Gerontologia. Rio de Janeiro: Guanabara Koogan, 2002.

CANINEU, P.R.; BASTOS, A. Transtorno cognitivo leve. Tratado de Geriatria e Gerontologia. Rio de Janeiro: Guanabara Koogan, 2002.

CÉSAR, I.D. Capacidade funcional em idosos institucionalizados. Dissertação (mestrado). Fortaleza, 2010.

CRESS, M.E.; BUCHNER, D.M.; QUESTAD, K.A. Exercise: effects on physical functional performance in independente older adults. Journal of Gerontology Advanced Biological Sciences Medical and Science. 1999.

DE VITTA, A. Atividade física e bem-estar na velhice. In A.L. Neri e S.A.Freire. (orgs.), E por falar em boa velhice. Campinas, 2000.

FREEDMAN, V.A.; AGREE, E.M.; MARTIN, L.G.; CORNMAN, J.C. Trends in the use of assistive technology and personal care for late-life disability, 1992-2001. Gerontologist. 2005.

GALLAHUE, D.L.; OZMUN, J.C. Compreendendo o desenvolvimento motor: bebês, crianças, adolescentes e adultos. São Paulo, 2005.

GARCIA, M. Uso de bengala necessita de prescrição médica, saiba como escolher a ideal. 2014. Disponível em <http://idmed.terra.com.br/>. Acesso em 22 dezembro 2014.

HAYFLICK, L. Como e porque envelhecemos. Rio de Janeiro: Campus, 1997

JANSSEN, I.; HEYMSFIELD, S.B.; WANG, Z.; ROSS, R. Skeletal muscle mass and distribution in 468 men and women aged 18-88 years. Journal of Applied Psysiology. 2000.

KAEMPF, G. Uso de muletas. 2014. Disponível em <www.gustavokaempf.com.br/>. Acesso em 15 dezembro 2014.

LEE, G.; OHNUMA, T.; CHONG, N.Y.; LEE, S. Walking Intent-Based Movement Control for JAIST Active Robotic Walker. IEEE transactions on systems, man, and cyberneticis: systems. 2014.

LIN, I.; WU, H. Activity Limitations, Use of Assistive Devices or Personal Help, and Well-Being: Variation by Education. The Journals of Gerontology. 2014. 


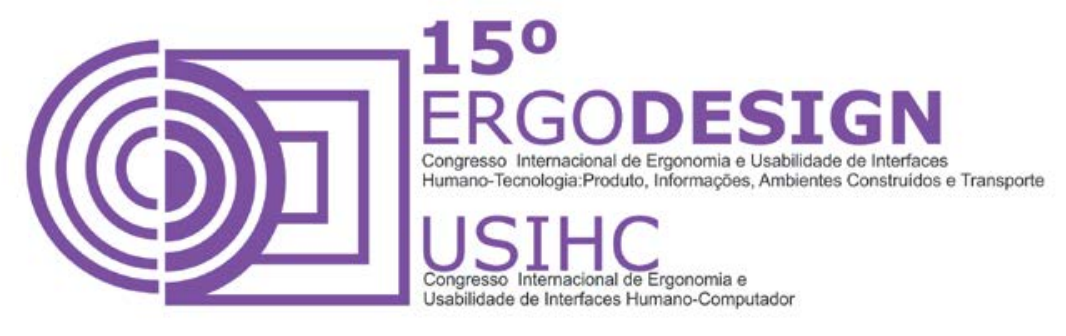

MANN, W.C.; HURREN, D.; TOMITA, M.; CHARVAT, B. An Analysis of Problems with Walkers Encountered by Elderly Persons. Physical \& Occupational Therapy in Geriatrics. 1995.

MATSUDO, S.M.; MATSUDO, V.K.R.; BARROS, T.L.N. Impacto do envelhecimento nas variáveis antropométricas, neuromotoras e metabólicas da aptidão física. Revista brasileira de ciência e movimento. Brasília, 2000.

MELLO, M.A.F; RAMOS, L.R. A necessidade de equipamentos de auto-ajuda e adaptações ambientais de idosos dependentes vivendo na comunidade. São Paulo, [tese]. São Paulo: UNIFESP, Escola de Medicina. 1999.

MINAYO, M.C.S.; O envelhecimento da população brasileira e os desafios para o setor saúde. Cadernos de Saúde Pública. Rio de Janeiro, 2012

NETTO, F.L.M.; Aspectos biológicos e fisiológicos do envelhecimento humano e suas implicações na saúde do idoso. Pensar a Prática. Goiânia, 2004.

ORGANIZAÇÃO PAN-AMERICANA DA SAÚDE. Envelhecimento ativo: uma política de saúde / World Health Organization. Brasília: Organização Pan-Americana da Saúde, 2005, 60p.

REEVES, N.D., MAGANARIS, C.N. E NARICI, M.V. Strength training alters the visco elastic properties of tendons in the elderly humans. Muscle \& Nerve. 2003.

SCHNEIDER, R. H.; MARCOLIN, D.; DALACORTE, R.R. Avaliação funcional de idosos. Scientia Medica. Porto Alegre, 2008.

SHEPHARD. R.J. Envelhecimento, atividade física e saúde. São Paulo: Phorte, 2003.

SOUZA, R.S.; MIRANDA, T.T.L.; SILVA, L.M.; MOREIRA, M.A.S.P.; LOPES, M.J.; MARQUES, M.C.M.P. Assisting technology: a study of social representations for health professionals. Revista de Pesquisa: Cuidado é Fundamental Online. Rio de Janeiro, 2011.

SPIRDUSO, W.W.; CRONIN, D.L. Exercise dose-response effects on quality of life and independent living in older adults. Medicine and Science in Sports and Exercise. 2001.

TEIXEIRA, M.H. Aspectos psicológicos da velhice. In A.L. Saldanha e C.P. Caldas (Ed.), Saúde do Idoso: a arte de cuidar. Rio de janeiro: Interciência, 2004.

THOMAS, B.; CONNELY, D.; RUDMAN, D.L. The impact and use of walkers among older adults: a pilot. Physical \& Occupational Therapy in geriatrics. 2008. 


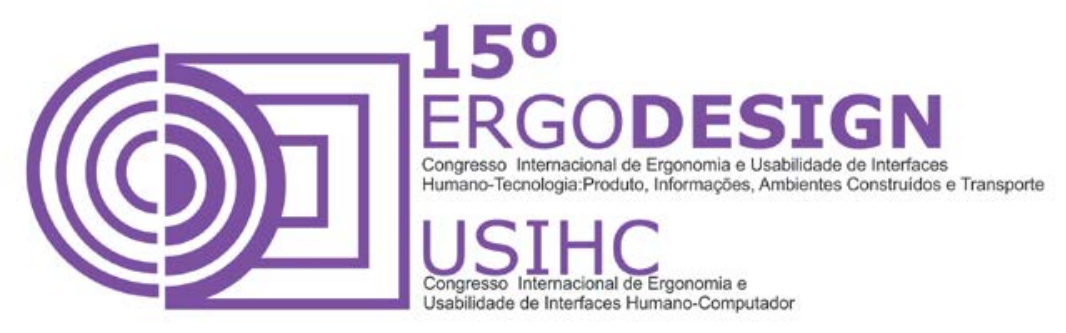

TOMITA, M.R.; MANN, W.C.; FRAAS, L.F.; STANTON, K.M. Predictors of the use of assistive devices that address physical impairments among community-based frail elders. Journal of Applied Gerontology. 2004.

WEINECK, J. Biologia do esporte. São Paulo: Manole, 1991.

ZIMERMAN, G.I. Velhice: aspectos biopsicossociais. Porto Alegre. Artes Médicas Sul, 2000. 